\title{
Domain-general and domain-specific aspects of temporal discounting in children with ADHD and autism spectrum disorders (ASD): A proof of concept study
}

\author{
Ellen Demurie ${ }^{1}$, Herbert Roeyers ${ }^{1}$, Dieter Baeyens ${ }^{2,3}, \&$ Edmund Sonuga-Barke ${ }^{1,4}$ \\ ${ }^{1}$ Department of Experimental Clinical and Health Psychology, Ghent University, Henri Dunantlaan 2, B-9000 \\ Ghent, Belgium \\ ${ }^{2}$ Code, Thomas More University College, Jozef De Bomstraat 11, B-2018 Antwerpen, Belgium \\ ${ }^{3}$ Department of Clinical Psychology, Katholieke Universiteit Leuven, Dekenstraat 2, B-3000 Leuven, Belgium \\ ${ }^{4}$ Developmental Brain-Behaviour Laboratory, University of Southampton, Highfield Campus
}

Southampton SO17 1BJ, UK

Note: This is an uncorrected version of an author's manuscript accepted for publication. Copyediting, typesetting, and review of the resulting proofs will be undertaken on this manuscript before final publication. During production and prepress, errors may be discovered that could affect the content.

Correspondence to Ellen Demurie, Ghent University, Department of Experimental Clinical and Health Psychology, Henri Dunantlaan 2, B-9000 Ghent, Belgium. Telephone: +32(0)9-2649411. Fax: +32(0)9-2646489.

Email: Ellen.Demurie@UGent.be.

Acknowledgements: We would like to thank all the participating children and adolescents, their parents and teachers. This research was supported by a grant from the Fund for Scientific Research - Flanders (Belgium) (FWO), awarded to the first author. 


\begin{abstract}
It has been shown that delayed consumable rewards are discounted to a higher degree than money, which has been referred to as the "domain effect". Until now the effects of reward type on temporal discounting (TD) have mainly been studied in adults. Although there is evidence that children with attention-deficit/hyperactivity disorder (ADHD) tend to show steeper TD of money than typically developing peers or children with autism spectrum disorders (ASD), it remains untested whether the domain effect is also seen in children with ADHD and ASD. To explore this we compared TD of children (8-16y) with ADHD, ASD and typically developing controls with five different reward types. Seventy-two participants with ADHD, 69 with ASD and 130 controls performed two hypothetical TD-tasks: a monetary TDtask and a TD-task with one of four alternative rewards (material rewards, rewarding activities, food, social rewards). TD was seen for all reward types, but the rate of discounting was steeper for food, praise and rewarding activities compared to money, and for food and praise compared to material rewards. For the ADHD and control groups, but not the ASD group, money and material rewards were equally highly discounted. High correlations between TD of money and of activities, food and material rewards were found. In conclusion, a domain effect was observed in typically developing children, as well as in children with ADHD or ASD, although the pattern was somewhat different for ASD children. Despite this domain effect, there is also evidence for a domain-general aspect in TD.
\end{abstract}

Keywords: temporal reward discounting, domain effect, ADHD, autism spectrum disorder, reward processing. 


\section{INTRODUCTION}

The decrease of subjective-reward value that occurs as a function of increasing delay is referred to as temporal discounting (TD; Critchfield \& Kollins, 2001). TD is a natural phenomenon that can be observed in both humans and animals (Green \& Myerson, 2004). TD is affected by a range of characteristics of individuals, such as IQ (de Wit, Flory, Acheson, McCloskey, \& Manuck, 2007; Olson, Hooper, Collins, \& Luciana, 2007) and age (Green, Fry, \& Myerson, 1994; Scheres et al., 2006). It appears altered in a number of mental health conditions (Ahn et al., 2011; Bickel \& Marsch, 2001; Reynolds, 2006). Steeper TD has been associated with impulsivity and hyperactivity (Barkley, Edwards, Laneri, Fletcher, \& Metevia, 2001; Critchfield \& Kollins, 2001; Scheres, Lee, \& Sumiya, 2008). Individuals with attention deficit/hyperactivity disorder (ADHD) show higher rates of TD of money, preferring immediate (monetary) rewards over larger delayed rewards to a greater extent than typically developing controls (Barkley et al., 2001; Demurie, Roeyers, Baeyens, \& Sonuga-Barke, 2012; Marco et al., 2009; Paloyelis, Asherson, Mehta, Faraone, \& Kuntsi, 2010; Scheres, Tontsch, Thoeny, \& Kaczkurkin, 2010; Sonuga-Barke, Taylor, Sembi, \& Smith, 1992; Wilson, Mitchell, Musser, Schmitt, \& Nigg, 2011). This has been regarded as evidence for a constitutional tendency to prefer immediate over delayed rewards even when the delayed reward is larger, which has been referred to as higher trait time preference in ADHD (Sonuga-Barke \& Fairchild, 2012). Other accounts highlight the role of altered emotional reactions to delay ("delay aversion"; Sonuga-Barke et al., 1992; Sonuga-Barke, Bitsakou, \& Thompson, 2010) or reduced executive control to inhibit choices for immediate tempting stimuli (Barkley, 1997). Although there is evidence that alterations in TD are associated with disorders other than ADHD, such as substance use disorder (Bickel \& Marsch, 2001) and depression (Ahn et al., 2011), there is also evidence of diagnostic specificity. For instance, TD of hypothetical monetary rewards was recently compared in children and adolescents with ADHD, autism spectrum disorders (ASD) and typically developing controls (Demurie et al., 2012). The ADHD group on average discounted rewards more steeply than the ASD group, who displayed the same degree of TD as their typically developing peers. These results are consistent with Antrop et al. (2006) who found 
that children with ASD did not show a preference for immediate monetary rewards over delayed rewards, like the children with ADHD did.

In general, there is evidence that the degree of TD varies as a function of intrinsic characteristics of rewards. Reward type (Charlton \& Fantino, 2008; Estle, Green, Myerson, \& Holt, 2007; König, 2009; Manwaring, Green, Myerson, Strube, \& Wilfley, 2011; Odum \& Rainaud, 2003) and reward magnitude (Scheres, Sumiya, \& Thoeny, 2010; Scheres, Tontsch, et al., 2010) both have an impact on TD. Studies in typically developing adults suggest that the extent to which a reinforcer can be considered to have "permanent" (i.e., typically with greater secondary-reinforcing properties) or "transient" (i.e., typically primary-rewarding properties) properties can have an effect on TD slopes. Individuals are willing to wait longer for permanent rewards, such as money, than for directly consumable rewards, such as food or drinks (i.e., transient rewards: Estle et al., 2007; Odum \& Rainaud, 2003), reflected by a steeper TD slope for transient compared to permanent rewards. This domainspecificity has been referred to as the "domain effect" (Charlton \& Fantino, 2008). Charlton and Fantino (2008) also found that food is discounted to a higher degree than money, but entertainment media, such as books and CDs, seem to show an intermediate level of TD steepness. They argue for a continuum of discounting rates influenced by several factors, such as primary- versus secondary-reinforcing qualities, possibility of immediate consumption, degree of perishability and satiability, etc. On the other hand, they found that individual discounting rates were highly consistent across reward types, depicted by positive inter-correlations between all possible pairs of rewards. Thus besides a domain-specific aspect, TD also has a domain-general aspect. The "domain effect" (Charlton \& Fantino, 2008) has mainly been studied in typically developing adult populations.

The evidence for increased TD in ADHD comes from TD tasks with both real and hypothetical monetary rewards (Barkley et al., 2001; Scheres, Tontsch, et al., 2010; Wilson et al., 2011) and over the short and the very long term (Barkley et al., 2001; Scheres, Tontsch, et al., 2010; but see Scheres et al., 2006 for a counter case). Until now, the domain effect of TD has not been investigated in an ADHD sample. Given the steeper TD slopes of money that can be observed in children and adolescents with ADHD compared to typically developing peers, the question arises whether the TD domain effect can be seen in these participants. Do children and adolescents with ADHD also show steeper TD slopes of primary, consumable and 
transient reinforcers compared to money? In real life, children are often rewarded with non-monetary rewards, such as social approval, rewarding activities, sweets, etc. If there is no evidence for a domain effect in ADHD children's TD slopes or a different domain-related pattern, then this might have implications for the choice of rewards in both experimental research and clinical practice. It is possible that individuals with ADHD respond differently to delayed money (and not to other delayed rewards) compared to other individuals because of its putative secondaryreinforcing properties, compared to more primary rewards. It is possible that a lower reinforcing effect of money in ADHD has an impact on the domain effect of TD in participants with ADHD, leading to a smaller difference in TD steepness of money compared to primary reinforcers. In the current study, we explored this hypothesis by examining TD to different reward types in groups of ADHD, ASD and control children and adolescents.

The current study is innovative as it is the first to investigate the TD "domain effect" in children with ADHD. We compared the relative impact of different reward types with more permanent reinforcing characteristics (money and material goods) versus rewards with transient reinforcing value (food, social praise, fun activities) on TD slopes. To get a view of the degree of disorder-specificity of potential abnormalities in TD processing in relation to the existence of the "domain effect", a clinical-contrast group of children with ASD was included in the current study. Similar to children with ADHD, children with ASD show deficits in some aspects of motivational processing (Berger, 2006; Dawson, Osterling, Rinaldi, Carver, \& McPartland, 2001; Koegel \& Mentis, 1985). In particular, studies suggest an insensitivity to social reward (Demurie, Roeyers, Baeyens, \& Sonuga-Barke, 2011; Garretson, Fein, \& Waterhouse, 1990; Geurts, Luman, \& van Meel, 2008; Schultz, 2005; Scott-Van Zeeland, Dapretto, Ghahremani, Poldrack, \& Bookheimer, 2010; but see Dichter, Richey, Rittenberg, Sabatino, \& Bodfish, 2012; Kohls et al., 2012). However, to date, while reward-related deficits are implicated in ASD, there is no evidence that these relate to the processing of delayed monetary rewards (Antrop et al., 2006; Demurie et al., 2012). Despite this evidence, it remains unknown whether children with ASD do differentiate between different types of delayed rewards and thus manifest a "domain effect" with regard to TD.

The current study was designed as an initial proof of concept study to establish whether or not the TD "domain effect" does or does not exist in children with and 
without ADHD or ASD. We had two main research questions. First, to get a picture of the domain-general aspect in TD, we investigated the interrelations between TD of money and the alternative rewards. We expected positive correlations between the different TD slopes, thus that subjects with a steeper monetary TD slope would also show steeper TD of the alternative rewards (Charlton \& Fantino, 2008). And second, do participants with ADHD and participants with ASD demonstrate the "domain effect" in TD, thus do they show less steep TD of money compared to the more transient, primary rewards, similar to the typically developing control group?

\section{MATERIAL AND METHODS}

\subsection{Participants}

Seventy-two children and adolescents with ADHD, 69 children and adolescents with ASD and 130 typically developing control children and adolescents participated in this study. All participants were between 8 and 16 years old and had an estimated full scale IQ (FSIQ) of 80 or more, measured with the Wechsler Intelligence Scale for Children - $3^{\text {rd }}$ edition (WISC-III; Wechsler, 1991). Four subtests (similarities, vocabulary, block design and picture arrangement) were administered. The sum of these subtest scores gives a reliable estimate of the FSIQ (Grégoire, 2005). Group characteristics are presented in Table 1. Groups did not differ in age, estimated FSIQ and sex ratio.

\section{Insert Table 1}

The participants with ADHD and ASD were all outpatients, recruited through rehabilitation centres, special school services, and other agencies specialized in the care of children with developmental disorders. All had previously been diagnosed with ADHD or ASD by a multidisciplinary team according to established criteria, as specified in the Diagnostic and Statistical Manual of Mental Disorders, fourth edition text revision (DSM-IV-TR; American Psychiatric Association, APA, 2000). Clinical diagnoses were confirmed with a validated measure: the Autism Diagnostic Interview, Revised (ADI-R; Lord, Rutter, \& Lecouteur, 1994), Autism Diagnostic Observational Schedule, Generic (ADOS-G; Lord et al., 2004) or Childhood Autism Rating Scale (CARS; Schopler, Reichler, Devellis, \& Daly, 1980) for ASD. The ADHD diagnosis was verified using the disruptive behaviour module (ADHD, oppositional defiant 
disorder, ODD, and conduct disorder, CD) of the Diagnostic Interview Scale for Children for DSM-IV (DISC-IV; Shaffer, Fisher, Lucas, Dulcan, \& Schwab-Stone, 2000). In the ADHD sample, 21 participants met the criteria for the predominantly inattentive subtype, four participants met the criteria for the predominantly hyperactive-impulsive subtype and 47 participants for the combined subtype, according to the DISC-IV. Children with ASD who had comorbid ADHD or clinical scores for teacher and parent ratings on the Disruptive Behavior Disorder rating scale (DBDRS; Pelham, Gnagy, Greenslade, \& Milich, 1992) were excluded from the sample. Participants on stimulant medication were asked to discontinue their medication at least 24 hours prior to testing. Fifty-two participants with ADHD were medicated with methylphenidate (MPH). In the ASD group, eight participants were medicated with MPH. Based on the results of the parent rated DBDRS, seven participants with ADHD and seven participants with ASD scored within the clinical range for ODD. Two participants with ADHD and three participants with ASD scored within the clinical range for CD.

Parents and teachers completed questionnaires to obtain a broad view on the child's overall functioning and possible comorbid symptoms. Parents completed the Child Behavior Checklist for ages 6-18 years (CBCL/6-18; Achenbach \& Rescorla, 2001), DBDRS, and Social Communication Questionnaire (SCQ; Rutter, Bailey, \& Lord, 2003). Teachers completed the Teacher's Report Form for ages 6-18 years (TRF/6-18; Achenbach \& Rescorla, 2001) and DBDRS.

\subsection{TD tasks}

Participants played two computerized tasks. In a first task, the children rated 30 pictures of one of the four alternative rewards (food, rewarding activities, social and material rewards) on a 7-point scale, ranging from 1 (not nice at all to receive this reward) through 4 (not nice, but also not annoying; neutral) to 7 (really nice) (see Figure 1). Participants were assigned to one of the four alternative reward conditions randomly. Each picture was shown three times, so that mean scores over the three ratings could be calculated. Based on this personal rating, a ranking of rewards was made and subjective values in percentages were calculated. The reward with a mean score of 4 (or the smallest value greater than 4 ) was equated with $0 \%$, and the reward with the highest mean score was equated with 100\%. By doing so, the subjective value of each reward for that participant was calculated, with $0 \%$ as neutral and $100 \%$ 
as most rewarding. Five of the rated rewards were selected as the alternative rewards in the second task.

Fig. 1 Example of a trial in the first task

In this second task, the actual Temporal Discounting (TD) task, participants were instructed to make repeated choices between a (hypothetical) small variable reward delivered immediately (now) and a large constant reward delivered after a variable delay (now, tomorrow, the day after tomorrow, 1 week, 2 weeks) (see Figure 2). This task consisted of two parts: a monetary TD task and an alternative reward TD task, showing the individual selection of pictures of the first task. Rewards in the monetary TD task were $0 €, 5 €, 10 €, 20 €$ and $30 €$, visualized as euro notes. Rewards in the alternative reward TD task were the rewards from the first task with subjective values (by approximation) $0 \%, 16.67 \%, 33.33 \%, 66.67 \%$ and $100 \%$. For 21 control participants, 21 participants with ADHD and 19 participants with ASD it was not possible to single out 5 alternative rewards with these subjective values, due to a lack of variability in rating scores. In these participants, only the monetary TD task has been administered.

Each small immediate reward was paired four times with every delay for the large reward, resulting in a total of 100 choice trials for each condition. Trials were administered in the same pseudo-random order to all participants. Left or right position of the delayed reward was balanced over trials. Participants responded by pressing button 1 when they preferred the left reward-delay combination and button 2 for the right reward-delay combination. The order of the two versions of the TD task was counterbalanced across subjects.

Fig. 2 Example of a trial for the material rewards and monetary TD tasks. Instruction: Which reward do you prefer, taking the delay into account?

Children were told that they would perform several computer tasks. Instructions were given both verbally and on the computer screen. While children read the instructions on-screen, the experimenter verbally explained them what they had to do. Children were told that they would see two pictures of rewards and that they had to choose which reward they would prefer to receive. However, below the picture of the reward they could see when they would receive this reward. Thus, they did not only 
have to choose what they liked the most, but they would also have to take into account how long they had to wait before receiving this reward. We reminded them that they had to take into account what they wanted and when they wanted it. There were no practice trials, but the experimenter repeated the instructions during the first three trials for all participants: e.g., "Do you prefer to receive 5 euro now or 20 euro in one week?". With this procedure, every child understood what he had to do during the TD task. It was also carefully explained that this was a hypothetical task, thus that they would not receive the rewards for real, but that they had to choose which rewarddelay combination they would prefer the most.

\subsection{Procedure}

For the administration of the computer tasks and the intelligence test, children and their parents were invited in the faculty of Psychology and Educational Sciences. To account for possible individual differences in perceived value of rewards, participants completed a questionnaire rating the subjective value of the monetary rewards used in the TD task using a 7-point Likert scale ranging from 1 (not valuable at all, no nice reward), over 4 (neutral), to 7 (very valuable, nice reward). All participants and their parents signed an informed consent form, prior to testing. At the end of the test session participants received a reward of 15 euro, but they were not aware of this during testing. The study was approved by the Ethical Committee of the faculty of Psychology and Educational Sciences of Ghent University.

\subsection{Data preprocessing}

Preprocessing of the data was based on procedures described by Scheres et al. (2006) and Critchfield and Kollins (2001). As a first step, subjective values for the delayed rewards were calculated for each delay. In order to determine the subjective values for the TD tasks, choice preferences were ordered based on delay duration and magnitude of the immediate reward. For each reward-delay combination, the answers were coded as "D" when the delayed reward was preferred and "I" when the immediate reward was preferred (see Table 2). Then, subjective values were determined for each delay by two independent raters based on the following procedure. The subjective value of the delayed reward was estimated by averaging the switch points in the ascending and descending choice sequences (Critchfield \& Kollins, 2001). These switch points are defined as the magnitudes of the small 
immediate reward for which the participant shows indifference in a choice against the large delayed reward (Scheres et al., 2006). In an ascending choice sequence, the large delayed reward is likely to be preferred initially (indicated by a " $\mathrm{D}$ " in Table 2), with preference shifting to the small immediate reward (indicated by an "I" in Table 2) as its size increases. In a descending choice sequence, the small immediate reward is likely to be preferred when the immediate reward has a large value, but preference shifts towards the large delayed reward as the size of the small immediate reward decreases (Critchfield \& Kollins, 2001). In Table 2, the determination of the subjective values is illustrated by an example of the monetary TD task. Agreement between the raters was high (mean kappa $=.99$, range $=.98-1.00)$. In rare cases of disagreement, the two ratings were compared and the disagreement was solved by correcting the mistake in the application of the rating rules.

The second step in the preprocessing of the data was to calculate the area under the curve (AUC) for the TD functions following the procedure described by Myerson, Green, and Warusawitharana (2001) and Scheres et al. (2006). First, subjective values and delays were normalized by expressing subjective values as proportions of the amount of the maximum delayed reward $(100 \%$ or $30 €)$ and expressing delays as proportions of the maximum delay ( 2 weeks). These normalized values were used as $x$ and $y$ coordinates to construct the discounting function, with delay on the $x$ axis and the subjective values on the $y$ axis. Then, vertical lines were drawn from each data point on the $x$ axis to determine four separate trapezoids. The area of each trapezoid is equal to $\left(x_{2}-x_{1}\right)\left[\left(y_{1}+y_{2}\right) / 2\right]$, where $x_{1}$ and $x_{2}$ are successive delays and $y_{1}$ and $y_{2}$ are the subjective values associated with these delays. Using this formula, the area of each trapezoid was calculated and subsequently the areas were summed, which resulted in the total AUC. The steeper the discounting function, the smaller the total AUC will be.

\section{Insert Table 2}

\subsection{Statistical analyses}

First, in order to validate the TD task we tested the extent to which TD was seen for each of the five reward types (money, activities, material, edible and social rewards). To do this, a series of repeated measures ANOVAs were used to investigate 
the effects of the within-subjects factor delay on TD slopes, irrespective of group. The dependent variable in these analyses was the normalized subjective value of the large reward under 4 delays: tomorrow, the day after tomorrow, 1 week and 2 weeks. The subjective value of the immediate large reward was not taken into account, as this was a constant value equal to 1 . TD occurred for all reward types although the pattern of effects was different (see Figure 3 for the TD slopes). For money and activities, the subjective value decreased as delay increased across each delay level: tomorrow $>$ day after tomorrow $>1$ week $>2$ weeks $(p<.001, p<.001$ and $p<.01$ for money; $p$ $<.01, p<.05$ and $p<.05$ for activities). For the material and edible rewards, discounting was seen between short delays compared to longer delays: tomorrow $=$ day after tomorrow $>1$ week $=2$ weeks $(p=1.00, p<.001$ and $p=1.00$ for the material rewards and $p=.48, p<.01$ and $p=.92$ for the edible rewards). Finally, for the social rewards, discounting was seen when comparing short and increasing delays: tomorrow $>1$ week $=2$ weeks $(p<.01$ and $p=0.55$ respectively $)$ and day after tomorrow $>2$ weeks $(p<.01)$.

As a second validity check, we tested whether, as in previous studies, ADHD participants had higher rates of TD for money than controls. All participants discounted the subjective value of money when delay-to-delivery increased. An ANOVA with group as factor and the AUC of the monetary TD task as the dependent variable showed a significant main effect of group, $F(2,268)=4.87, p<.01, \eta_{\mathrm{p}}{ }^{2}=$ .04. Post hoc analyses (Bonferroni) showed that AUC of the ADHD group was significantly lower than AUC of the controls $(p<.01)$ and marginally significantly lower than that of the ASD group $(p=.09)$. The AUC of participants with ASD was not significantly different from the AUC of controls $(p=1.00)$. Group means are presented in Table 3. Group differences in TD do not seem to be due to differences in subjective value of the monetary reward amounts. Reward amounts $(0,5,10,20$ and $30 €)$ were rated significantly different in value from each other, $F(4,257)=567.38, p$ $<.001, \eta_{\mathrm{p}}^{2}=.90$, with an increase in subjective value for each increase in reward value $(p<.001$ for all contrasts). There were no significant group differences in perceived value of monetary rewards, $F(2,260)=.45, p=.64, \eta_{\mathrm{p}}{ }^{2}=.00$.

Fig. 3 TD slopes of the different TD tasks 
To answer our research questions, first, correlations between the AUC of money and the separate alternative rewards have been calculated to get a picture of the domain-general aspect of TD. Second, the domain effect of TD was investigated in the three groups with an ANOVA to compare the AUC's of the alternative TD tasks and a series of repeated measures analyses to compare the AUC's of the monetary TD task versus the separate alternative reward TD tasks. Group was included as a between-subjects factor in these analyses.

Age and IQ were not correlated with TD measures for any type of reward and did not differ between the different groups working for different types of rewards. Therefore, in order to retain as much statistical power as possible, we did not include these variables as covariates in the analyses.

\section{RESULTS}

\subsection{Are the AUC's of different reward types correlated?}

AUC of money correlated significantly with the AUC of activities, $r(53)=.49$, $p<.001$, material rewards, $r(50)=.51, p<.001$, and food, $r(52)=.41, p<.01$, but not with the AUC of social rewards, $r(55)=.17, p=.22$. Correlations did not change when group was taken into account: partial correlations: $r(50)=.49, p<.001 ; r(47)=$ $.53, p<.001 ; r(49)=.41, p<.01 ;$ and $r(52)=.18, p=.19$ respectively. The pattern of correlations was very similar for the three participating groups (data available from first author).

\subsection{Exploration of the TD domain effect: Are different rewards discounted at different rates and are there any group differences?}

When the AUC's of the 4 alternative rewards were compared, a significant main effect of alternative TD condition, $F(3,198)=4.26, p<.01, \eta_{\mathrm{p}}{ }^{2}=.06$, was found. The AUC for the more permanent material rewards was significantly higher than the AUC's for the (transient) edible and social rewards ( $p<.01$ and $p<.05$ respectively). The remaining AUC's were not significantly different (material rewards versus activities: $p=.13$, other contrasts: $p=1.00$, see Figure 3 for the TD slopes). No main or interaction effect of group was found. 
Furthermore the AUC's of money and the separate alternative rewards were compared. The monetary AUC was significantly higher than the AUC of rewarding activities, $F(1,50)=20.39, p<.001, \eta_{\mathrm{p}}{ }^{2}=.29$, edible rewards, $F(1,49)=20.01, p$ $<.001, \eta_{\mathrm{p}}^{2}=.29$, and social rewards, $F(1,52)=21.96, p<.001, \eta_{\mathrm{p}}{ }^{2}=.30$, for all participants. No main or interaction effects of group were found for these three comparisons. Between the AUC's of monetary and material rewards, however, no significant difference was found, $F(1,47)=2.82, p=.10, \eta_{\mathrm{p}}^{2}=.06$, but an interaction effect between reward type and group, $F(2,47)=4.08, p<.05, \eta_{\mathrm{p}}{ }^{2}=.15$, emerged. Only in the ASD group, but not in the control and ADHD groups, a significant difference between the AUC of material and monetary rewards could be observed, $F(1,10)=9.99, p<.05, \eta_{\mathrm{p}}^{2}=.50$, with a steeper TD slope for material rewards.

\section{Insert Table 3}

Fig. 4 AUC values of the different TD tasks

\section{DisCUSSION}

The current study was the first to explore the domain-general and domainspecific aspects of TD in participants with ADHD, ASD and their typically developing peers on hypothetical TD tasks across different reward types.

First of all, strong positive correlations between TD of money, on the one hand, and activities, food and material rewards, on the other hand, could be observed in the hypothetical TD tasks. Children and adolescents (with and without developmental disabilities) who discount money at a high rate tend to discount activities, food or material rewards at a high rate too. Notwithstanding domain-specificity in TD, there is also evidence for a domain-general aspect of TD (see also Charlton \& Fantino, 2008; Tsukayama \& Duckworth, 2010). As an exception, TD of social rewards did not correlate with TD of money. It is possible that intertemporal decisions with regard to social rewards differ from decisions on more tangible rewards. However, Tayler, Arantes, and Grace (2009) have compared TD of hypothetical monetary rewards and hypothetical relationship outcomes. Although the discounting rates differed in the two domains, participants seemed to make similar intertemporal choices about 
relationships as when they were deciding about monetary outcomes. In the current study, the social rewards were differently operationalized as compliments by meaningful persons, medals that could be won, smiling faces, etc. The specific nature of the (social) reward seems to be important with respect to TD.

Second, in general, TD slopes were steeper for transient rewards with stronger putative primary-reinforcing properties (rewarding activities, edible and social rewards) compared to money, with material rewards appearing more like money than the other rewards. Thus, the steepness of a TD slope seemed to be dependent on the specific nature of rewards for all participants (Estle et al., 2007; König, 2009; Odum \& Rainaud, 2003; Tayler et al., 2009), which is in line with the "domain effect". It is known that consumable rewards are discounted more steeply than money (Estle et al., 2007; Odum \& Rainaud, 2003). The current study confirms this and extends the finding to activities and social rewards. Rewarding activities, edible and social rewards are transient and cannot be collected, while money with its putative secondary-reinforcing properties has more permanent value. It can be collected and can be used to buy other goods (Estle et al., 2007). Compared to the other alternative rewards, material rewards also seemed to have a special status. No difference between TD of monetary and material rewards could be found in the control and ADHD groups. Moreover, material rewards were discounted less steeply than edible and social rewards. Just like money, material goods retain their value, since they are not perishable, they can be sold, etc. The tangible and collectable nature of a material reward seems to have an impact on its subjective value when reward delivery is delayed, providing it with a longer lasting rewarding effect. Evidence for the "domain effect" could thus be found in the three participating groups, although the pattern was somewhat different for the ASD participants. For activities, food and social rewards versus money, there was no interaction with group, indicating that all participants showed steeper TD for these transient rewards compared to money and thus a clear "domain effect". For material rewards however, an interaction between reward type and group indicated that only ADHD and control participants showed similar TD steepness for money and material rewards. The ASD group discounted material rewards to a greater extent than money. One possible explanation is that the preferred material goods have an especially potent primary-reinforcing power because of specific preoccupations. If children with ASD rated objects for which they have preoccupations in the first computer task as their preferred rewards, then this might 
have led to steeper TD of these material rewards compared to money. Future studies should further investigate this hypothesis.

In general, we might conclude that the "domain effect" is a characteristic that can be observed in both clinical and non-clinical groups, but the pattern might differ according to personal preferences and specific problems one deals with. In line with this suggestion, Rasmussen, Lawyer, and Reilly (2010) found that percentage of body fat predicted TD of hypothetical food, but not money. Thus, the "domain effect", although a robust phenomenon, seems to be influenced by individual factors too.

Our results have significance from both theoretical and practical perspectives. From a scientific perspective, the fact that the ADHD sample does show a domain effect in TD (less steep TD for money compared to transient rewards) despite the fact that they have steeper TD slopes for money seems to suggest that they do understand the value of this secondary reinforcer. In line with this, subjective values of the monetary rewards were rated similarly (in a questionnaire) in the three groups. On the other hand we found steeper TD slopes for money in this sample, which might be due to (1) an overall stronger sensitivity to delay before reward (Sonuga-Barke \& Fairchild, 2012) - implying that TD slopes of all reward types should be steeper compared to controls - or to (2) the fact that ADHD individuals respond differently to money compared to other rewards. This might be due to a less well-developed model of monetary-reward value, failing to distinguish the secondary-reinforcing value of money as opposed to other more primary and transient rewards. This would imply that only monetary TD slopes are steeper compared to typically developing peers. However, only by investigating group differences in TD with different reward types, one can test these hypotheses. However, we were not able to do this in the current paper given limited sample sizes for the separate non-monetary reward types and thus put it forward as a challenge for future studies. From a practical point of view, although using money in TD tasks has the advantages of being easy to administer and accurately quantifiable (Hinvest \& Anderson, 2010), the ecological validity and generalizability of the research results with monetary TD tasks may be limited because we do not know whether these generalize to other reward types. The finding of a domain-general aspect of TD however suggests that there might be at least some degree of generalizability. Moreover, in the context of education and parenting, rewards with transient and primary-reinforcing qualities are more frequently used. To understand TD (and ADHD and ASD) in the real world, we investigated the domain 
effect of TD by comparing the effects of delay-to-delivery for different reward types with permanent and stronger secondary-reinforcing characteristics versus those with more transient value, such as enjoyable activities and tasty food. This study is only a first step in the direction of a more comprehensive understanding of how TD translates into daily decision making.

Based on our results, we can formulate some implications and directions for future research. First, although TD tasks may not be used diagnostically, hypothetical monetary TD tasks can be used in assessment procedures to shed light on the functional impairments of children with ADHD with regard to delay aversion and to strive for the best individually-tailored treatment planning (Karalunas \& HuangPollack, 2011). Second, it would be interesting to investigate the neural basis of TD and its development in both clinical (ADHD and ASD) and typically developing samples with neuro-imaging techniques, such as fMRI or event-related potentials.

Although the findings above are innovative and enlarge our insight into TD of different reward types in children and adolescents with and without developmental problems, there are some limitations that should be acknowledged when interpreting the results of this study. First, this study was not powered statistically to test for between-group differences in TD slopes of alternative rewards, given the limited sample sizes of the separate non-monetary reward conditions. Future studies could include larger samples per alternative reward or administer different alternative reward TD tasks to all subjects.

Second, in the current study we did not screen for receptive language problems, which are often seen in ASD (Stefanatos \& Baron, 2011). Language problems might affect the degree of understanding the task instructions and can thus alter test results. All participants in the current study seemed to understand the task instructions and were able to engage in a mutual conversation. Nevertheless, it might be interesting to take language skills into account in future TD studies.

Third, although the alternative rewards for the TD task were selected based on individual ratings, we did not directly compare the subjective values of money and the alternative rewards for each subject. Tsukayama and Duckworth (2010) suggest that steeper TD slopes can be found for rewards that are desired and enjoyed more than others. In their opinion, temptation explains at least partly domain-specific TD. A person who is really tempted by candies might show steeper TD for candies compared to a less preferred reward. However, Chapman (1996) found that domain-specificity 
of discounting rates persists even after matching the outcomes for utility and subjective value. Nevertheless, future research should take temptation toward the presented rewards and individual preferences for different reward types into account.

Fourth, the hypothetical TD tasks were not validated in a pre-study. We performed two validity checks in the current study. First of all, in line with previous studies in adult samples (Estle et al., 2007; König, 2009; Odum \& Rainaud, 2003; Tayler et al., 2009), we found subjective values of all rewards to decrease when reward-to-delivery increased. It seems that TD can be seen with a range of different hypothetical rewards in child and adolescent samples. These results open possibilities for future research as they point out that different hypothetical rewards can be employed in TD tasks, adapted for use in children and adolescents with and without developmental disorders. Second, TD slopes of money were compared in the three participating groups. We found a difference in TD of monetary rewards between children and adolescents with ADHD on the one hand and typically developing children and adolescents (and a trend for the children and adolescents with ASD) on the other hand. Steeper TD slopes of monetary rewards in ADHD are a robust finding (Barkley et al., 2001; Demurie et al., 2012; Paloyelis et al., 2010; Scheres, Tontsch, et al., 2010; Wilson et al., 2011) and have been linked to the trait time preference (Sonuga-Barke \& Fairchild, 2012) and delay aversion (Sonuga-Barke et al., 1992) attributed to children with ADHD. Functional magnetic resonance imaging research shows that more impulsive individuals show greater neural deactivations to the delay of future rewards in the mesial and dorsolateral prefrontal cortex and the posterior parietal cortex, suggesting that they show greater (negative) sensitivity to longer delays before delivery of future rewards (Ballard \& Knutson, 2009). The delay related characteristics of monetary reward processing may be considered as a specific characteristic of ADHD. Steep TD of monetary rewards seems to be a trait-like characteristic that is at least partly attributable to genetic differences in the dopaminergic system (Peters \& Büchel, 2011). Participants with ASD did not show a steeper monetary TD slope compared to typically developing participants. These results are consistent with those of Antrop et al. (2006) and Demurie et al. (2012) who found no evidence for delay aversive behaviours or steeper monetary TD in ASD. Unfortunately, due to limited sample sizes and power issues, group effects could not be investigated for the alternative rewards.

Fifth, surprisingly age and IQ did not correlate with the AUC values. However, 
TD has been found to be associated with these individual characteristics by many others. First, age effects on TD have been found consistently in both real and hypothetical monetary TD tasks (Green et al., 1994; Olson et al., 2007; Scheres et al., 2006; Steinberg et al., 2009). However, Reynolds (2006) suggests that real TD tasks may be better suited for use with children because the choices are less abstract compared to hypothetical TD tasks. It is possible that a real TD task would have been more sensitive to age effects. Future studies should further investigate this issue. Second, in their review, Shamosh and Gray (2008) reported that even in hypothetical TD tasks correlations between TD and intelligence have been observed. Although the relation between TD and intelligence is often regarded as well established, Barkley et al. (2001) also found no effect of IQ level (average or below versus above average) on TD. Maybe our null finding can be related to our measure of IQ. We estimated full scale IQ on the basis of four subscales of the WISC-III, but we were not able to extract an estimate of the verbal and performal IQ. Olson et al. (2007) suggested that the association between IQ and TD is driven solely by verbal aspects of intelligence. It would have been better if we had included (estimates of) both performance and verbal, as well as full scale IQ scores.

\section{Conclusions}

In conclusion, in the current study TD slopes of transient rewards (food, praise and rewarding activities) were steeper than the TD slopes of more permanent rewards (money and material rewards). This specific domain effect pattern could be observed in typically developing and ADHD participants. Participants with ASD showed a slightly different domain effect with a less steep TD slope of money compared to all alternative rewards (thus also for material rewards). Furthermore, individual discounting rates were consistent across reward types, as high interrelations between TD of money on the one hand and activities, food and material rewards on the other hand could be found. We hope that the results of this study can be of help in evolving to a better understanding of the effects of delay on daily decision-making processes. 


\section{Highlights}

- Domain-specific and domain-general temporal discounting (TD) was investigated in children with ADHD or ASD.

- TD was seen for all reward types.

- TD rate differed between transient and more permanent rewards.

- A domain effect was observed in controls, children with ADHD and those with ASD.

- Evidence for a domain-general aspect in TD was also found. 


\section{REFERENCES}

Achenbach, T.M., \& Rescorla, L.A. (2001). Manual for the ASEBA school-age forms and profiles. Burlington: University of Vermont.

Ahn, W.Y., Rass, O., Fridberg, D.J., Bishara, A.J., Forsyth, J.K., Breier, A., ... O'Donnell, B.F. (2011). Temporal discounting of rewards in patients with bipolar disorder and schizophrenia. Journal of Abnormal Psychology, 120(4), 911-921. doi: 10.1037/a0023333

American Psychiatric Association (2000). Diagnostic and Statistical Manual of Mental Disorders (4th edition, text revision). Washington, DC: APA.

Antrop, I., Stock, P., Verté, S., Wiersema, J.R., Baeyens, D., \& Roeyers, H. (2006). ADHD and delay aversion: The influence of non-temporal stimulation on choice for delayed rewards. Journal of Child Psychology and Psychiatry, 47(11), 1152-1158. doi: 10.1111/j.1469-7610.2006.01619.x

Ballard, K., \& Knutson, B. (2009). Dissociable neural representations of future reward magnitude and delay during temporal discounting. NeuroImage, 45(1), 143-150. doi: 10.1016/j.neuroimage.2008.11.004

Barkley, R.A. (1997). Behavioral inhibition, sustained attention, and executive functions: Constructing a unifying theory of ADHD. Psychological Bulletin, 121(1), 65-94. doi: 10.1037//0033-2909.121.1.65

Barkley, R.A., Edwards, G., Laneri, M., Fletcher, K., \& Metevia, L. (2001). Executive functioning, temporal discounting, and sense of time in adolescents with attention deficit hyperactivity disorder (ADHD) and oppositional defiant disorder (ODD). Journal of Abnormal Child Psychology, 29(6), 541-556. doi: 10.1023/A:1012233310098

Berger, M. (2006). A model of preverbal social development and its application to social dysfunctions in autism. Journal of Child Psychology and Psychiatry, 47, 338-371. doi: 10.1111/j.1469-7610.2005.01586.x

Bickel, W.K., \& Marsch, L.A. (2001). Toward a behavioral economic understanding of drug dependence: Delay discounting processes. Addiction, 96(1), 73-86. doi: $10.1080 / 09652140020016978$

Chapman, G.B. (1996). Temporal discounting and utility for health and money. Journal of Experimental Psychology: Learning, Memory and Cognition, 22, 
771-791. doi: 10.1037//0278-7393.22.3.771

Charlton, S.R., \& Fantino, E. (2008). Commodity specific rates of temporal discounting: Does metabolic function underlie differences in rates of discounting? Behavioural processes, 77(3), 334-342. doi: 10.1016/j.beproc.2007.08.002

Critchfield, T.S., \& Kollins, S.H. (2001). Temporal discounting: Basic research and the analysis of socially important behavior. Journal of Applied Behavior Analysis, 34(1), 101-122. doi: 10.1901/jaba.2001.34-101

Dawson, G., Osterling, J., Rinaldi, J., Carver, L., \& McPartland, J. (2001). Brief report: Recognition memory and stimulus-reward associations: Indirect support for the role of ventromedial prefrontal dysfunction in autism. Journal of Autism and Developmental Disorders, 31, 337-341. doi: 10.1023/A:1010751404865

de Wit, H., Flory, J.D., Acheson, A., McCloskey, M., \& Manuck, S.B. (2007). IQ and nonplanning impulsivity are independently associated with delay discounting in middle-aged adults. Personality and Individual Differences, 42(1), 111-121. doi: 10.1016/j.paid.2006.06.026

Demurie, E., Roeyers, H., Baeyens, D., \& Sonuga-Barke, E. (2011). Common alterations in sensitivity to type but not amount of reward in ADHD and autism spectrum disorders. Journal of Child Psychology and Psychiatry, 52(11), 11641172. doi: 10.1111/j.1469-7610.2010.02374.x

Demurie, E., Roeyers, H., Baeyens, D., \& Sonuga-Barke, E. (2012). Temporal discounting of monetary rewards in children and adolescents with ADHD and autism spectrum disorders. Developmental Science, 15(6), 791-800. doi: 10.1111/j.1467-7687.2012.01178.x

Dichter, G.S., Richey, J.A., Rittenberg, A.M., Sabatino, A., \& Bodfish, J.W. (2012). Reward circuitry function in autism during face anticipation and outcomes. Journal of Autism and Developmental Disorders, 42(2), 147-160. doi: 10.1007/s10803-011-1221-1.

Estle, S.J., Green, L., Myerson, J., \& Holt, D.D. (2007). Discounting of monetary and directly consumable rewards. Psychological Science, 18(1), 58-63. doi: 10.1111/j.1467-9280.2007.01849.x

Garretson, H.B., Fein, D., \& Waterhouse, L. (1990). Sustained attention in children with autism. Journal of Autism and Developmental Disorders, 20, 101-114. doi: 10.1007/BF02206860 
Geurts, H.M., Luman, M., \& van Meel, C. (2008). What's in a game: The effect of social motivation on interference control in boys with ADHD and autism spectrum disorders. Journal of Child Psychology and Psychiatry, 49, 848-857. doi: 10.1111/j.1469-7610.2008.01916.x

Green, L., \& Myerson, J. (2004). A discounting framework for choice with delayed and probabilistic rewards. Psychological Bulletin, 130(5), 769-792. doi: 10.1037/0033-2909.130.5.769

Green, L., Fry, A.F., \& Myerson, J. (1994). Discounting of delayed rewards: A lifespan comparison. Psychological Science, 5(1), 33-36. doi: 10.1111/j.14679280.1994.tb00610.x

Grégoire, J. (2005). L'évaluation clinique de l'intelligence de l'enfant. Théorie et pratique du WISC-III, troisième édition. Sprimont : Mardaga.

Hinvest, N.S., \& Anderson, I.M. (2010). The effects of real versus hypothetical reward on delay and probability discounting. Quarterly Journal of Experimental Psychology, 63(6), 1072-1084. doi: 10.1080/17470210903276350

Karalunas, S.L., \& Huang-Pollock, C.L. (2011). Examining relationships between executive functioning and delay aversion in attention deficit hyperactivity disorder. Journal of Clinical Child and Adolescent Psychology, 40(6), 837-847. doi: 10.1080/15374416.2011.614578

Koegel, R.L., \& Mentis, M. (1985). Motivation in childhood autism: Can they or won't they? Journal of Child Psychology and Psychiatry, 26, 185-191. doi: 10.1111/j.1469-7610.1985.tb02259.x

Kohls, G., Schulte-Rüther, M., Nehrkorn, B., Müller, K., Fink, G.R., Kamp-Becker, I., .. Konrad, K. (2012). Reward system dysfunction in autism spectrum disorders. Social Cognitive and Affective Neuroscience, Epub ahead of print.

König, C.J. (2009). A generalizability study of time discounting: Some people react more strongly to domain differences than others. Swiss Journal of Psychology, 68(1), 43-50. doi: 10.1024/1421-0185.68.1.43

Lord, C., Risi, S., Lambrecht, L., Cook, E.H., Leventhal, B.L., DiLavore, P.C., ... Rutter M. (2004). The Autism Diagnostic Observation Schedule-Generic: A standard measure of social and communication deficits associated with the spectrum of autism. Journal of Autism and Developmental Disorders, 30, 205223. doi: $10.1023 / \mathrm{A}: 1005592401947$ 
Lord, C., Rutter, M., \& Lecouteur, A. (1994). Autism Diagnostic Interview-Revised: A revised version of a diagnostic interview for caregivers of individuals with possible pervasive developmental disorders. Journal of Autism and Developmental Disorders, 24, 659-685. doi: 10.1007/BF02172145

Manwaring, J.L., Green, L., Myerson, J., Strube, M.J., \& Wilfley, D.E. (2011). Discounting of various types of rewards by women with and without binge eating disorder: Evidence for general rather than specific differences. The Psychological Record, 61, 561-582.

Marco, R., Miranda, A., Schlotz, W., Melia, A., Mulligan, A., Müller, U., ... SonugaBarke, E.J.S. (2009). Delay and reward choice in ADHD: An experimental test of the role of delay aversion. Neuropsychology, 23(3), 367-380. doi: $10.1037 / \mathrm{a} 0014914$

Myerson, J., Green, L., \& Warusawitharana, M. (2001). Area under curve as a measure of discounting. Journal of the Experimental Analysis of Behaviour, 76, 235-243. doi: 10.1901/jeab.2001.76-235

Odum, A.L., \& Rainaud, C.P. (2003). Discounting of delayed hypothetical money, alcohol, and food. Behavioural Processes, 64(3), 305-313. doi: 10.1016/S03766357(03)00145-1

Olson, E.A., Hooper, C.J., Collins, P., \& Luciana, M. (2007). Adolescents' performance on delay and probability discounting tasks: Contributions of age, intelligence, executive functioning, and self-reported externalizing behavior. Personality and Individual Differences, 43(7), 1886-1897. doi: 10.1016/j.paid.2007.06.016

Paloyelis, Y., Asherson, P., Mehta, M. A., Faraone, S. V., \& Kuntsi, J. (2010). DAT1 and COMT effects on delay discounting and trait impulsivity in male adolescents with attention deficit/hyperactivity disorder and healthy controls. Neuropsychopharmacology, 35(12), 2414-2426. doi: 10.1038/npp.2010.124

Pelham, W.E., Gnagy, E.M., Greenslade, K.E., \& Milich, R. (1992). Teacher ratings of DSM-III-R symptoms for disruptive behavior disorders. Journal of the American Academy of Child and Adolescent Psychiatry, 31, 210-218. doi: 10.1097/00004583-199203000-00006

Peters, J., \& Büchel, C. (2011). The neural mechanisms of inter-temporal decisionmaking: Understanding variability. Trends in Cognitive Sciences, 15(5), 227239. doi: 10.1016/j.tics.2011.03.002 
Rasmussen, E.B., Lawyer, S.R., \& Reilly, W. (2010). Percent body fat is related to delay and probability discounting for food in humans. Behavioural processes, 83(1), 23-30. doi: 10.1016/j.beproc.2009.09.001

Reynolds, B. (2006). A review of delay-discounting research with humans: Relations to drug use and gambling. Behavioural Pharmacology, 17(8), 651-667. doi: 10.1097/FBP.0b013e3280115f99

Rutter, M., Bailey, A., \& Lord, C. (2003). Social Communication Questionnaire. California: Western Psychological Services.

Scheres, A., Dijkstra, M., Ainslie, E., Balkan, J., Reynolds, B., Sonuga-Barke, E., \& Castellanos, F. X. (2006). Temporal and probabilistic discounting of rewards in children and adolescents: Effects of age and ADHD symptoms. Neuropsychologia, 44(11), 2092-2103. doi: 10.1016/j.neuropsychologia.2005.10.012

Scheres, A., Lee, A., \& Sumiya, M. (2008). Temporal reward discounting and ADHD: Task and symptom specific effects. Journal of Neural Transmission, 115(2), 221-226. doi: 10.1007/s00702-007-0813-6

Scheres, A., Sumiya, M., \& Thoeny, A. L. (2010). Studying the relation between temporal reward discounting tasks used in populations with ADHD: A factor analysis. International Journal of Methods in Psychiatric Research, 19(3), 167176. doi: $10.1002 / \mathrm{mpr} .309$

Scheres, A., Tontsch, C., Thoeny, A. L., \& Kaczkurkin, A. (2010). Temporal reward discounting in attention-deficit/hyperactivity disorder: The contribution of symptom domains, reward magnitude, and session length. Biological Psychiatry, 67(7), 641-648. doi: 10.1016/j.biopsych.2009.10.033

Schopler, E., Reichler, R.J., Devellis, R.F., \& Daly, K. (1980). Toward objective classification of childhood autism: Childhood autism rating-scale (CARS). Journal of Autism and Developmental Disorders, 10, 91-103. doi: 10.1007/BF02408436

Schultz, R.T. (2005). Developmental deficits in social perception in autism: The role of the amygdala and fusiform face area. International Journal of Developmental Neuroscience, 23, 125-141. doi: 10.1016/j.ijdevneu.2004.12.012

Scott-Van Zeeland, A.A., Dapretto, M., Ghahremani, D.G., Poldrack, R.A., \& Bookheimer, S.Y. (2010). Reward processing in autism. Autism Research, 3, 115. doi: 10.1002/aur.122 
Shaffer, D., Fisher, P., Lucas, C.P., Dulcan, M.K., \& Schwab-Stone, M.E. (2000). NIMH Diagnostic Interview Schedule for Children Version IV: Description, differences from previous versions, and reliability of some common diagnoses. Journal of the American Academy of Child and Adolescent Psychiatry, 39, 2838. doi: 10.1097/00004583-200001000-00014

Shamosh, N.A., \& Gray, J.R. (2008). Delay discounting and intelligence: A metaanalysis. Intelligence, 36, 289-305. doi: 10.1016/j.intell.2007.09.004

Sonuga-Barke, E.J.S., \& Fairchild, G. (2012). Neuroeconomics of attentiondeficit/hyperactivity disorder: Differential influences of medial, dorsal and ventral prefrontal brain networks on sub-optimal decision-making? Biological Psychiatry, Epub ahead of print. doi: 10.1016/j.biopsych.2012.04.004

Sonuga-Barke, E.J.S., Bitsakou, P., \& Thompson, M. (2010). Beyond the dual pathway model: Evidence for the dissociation of timing, inhibitory, and delayrelated impairments in attention-deficit/hyperactivity disorder. Journal of the American Academy of Child and Adolescent Psychiatry, 49(4), 345-355. doi: 10.1016/j.jaac.2009.12.018

Sonuga-Barke, E.J.S., Taylor, E., Sembi, S., \& Smith, J. (1992). Hyperactivity and delay aversion: I. The effect of delay on choice. Journal of Child Psychology and Psychiatry and Allied Disciplines, 33(2), 387-398. doi: 10.1111/j.14697610.1992.tb00874.x

Stefanatos, G.A., \& Baron, I.S. (2011). The ontogenesis of language impairment in autism: A neuropsychological perspective. Neuropsychology Review, 21, 252270. doi: 10.1007/s11065-011-9178-6

Steinberg, L., Graham, S., O’Brien, L., Woolard, J., Cauffman, E., \& Banich, M. (2009). Age differences in future orientation and delay discounting. Child Development, 80, 28-44. doi: 10.1111/j.1467-8624.2008.01244.x

Tayler, S., Arantes, J., \& Grace, R. C. (2009). Temporal discounting for monetary and close relationship outcomes. Personal Relationships, 16(3), 385-400. doi: 10.1111/j.1475-6811.2009.01229.x

Tsukayama, E., \& Duckworth, A.L. (2010). Domain-specific temporal discounting and temptation. Judgment and Decision Making, 5(2), 72-82. Retrieved from http://www.libsearch.com/view/1173102 
Wechsler, D. (1991). Wechsler Intelligence Scale for Children (3rd ed.). New York: Psychological Corporation.

Wilson, V. B., Mitchell, S. H., Musser, E. D., Schmitt, C. F., \& Nigg, J. T. (2011). Delay discounting of reward in ADHD: Application in young children. Journal of Child Psychology and Psychiatry, 52(3), 256-264. doi: 10.1111/j.14697610.2010.02347.x 


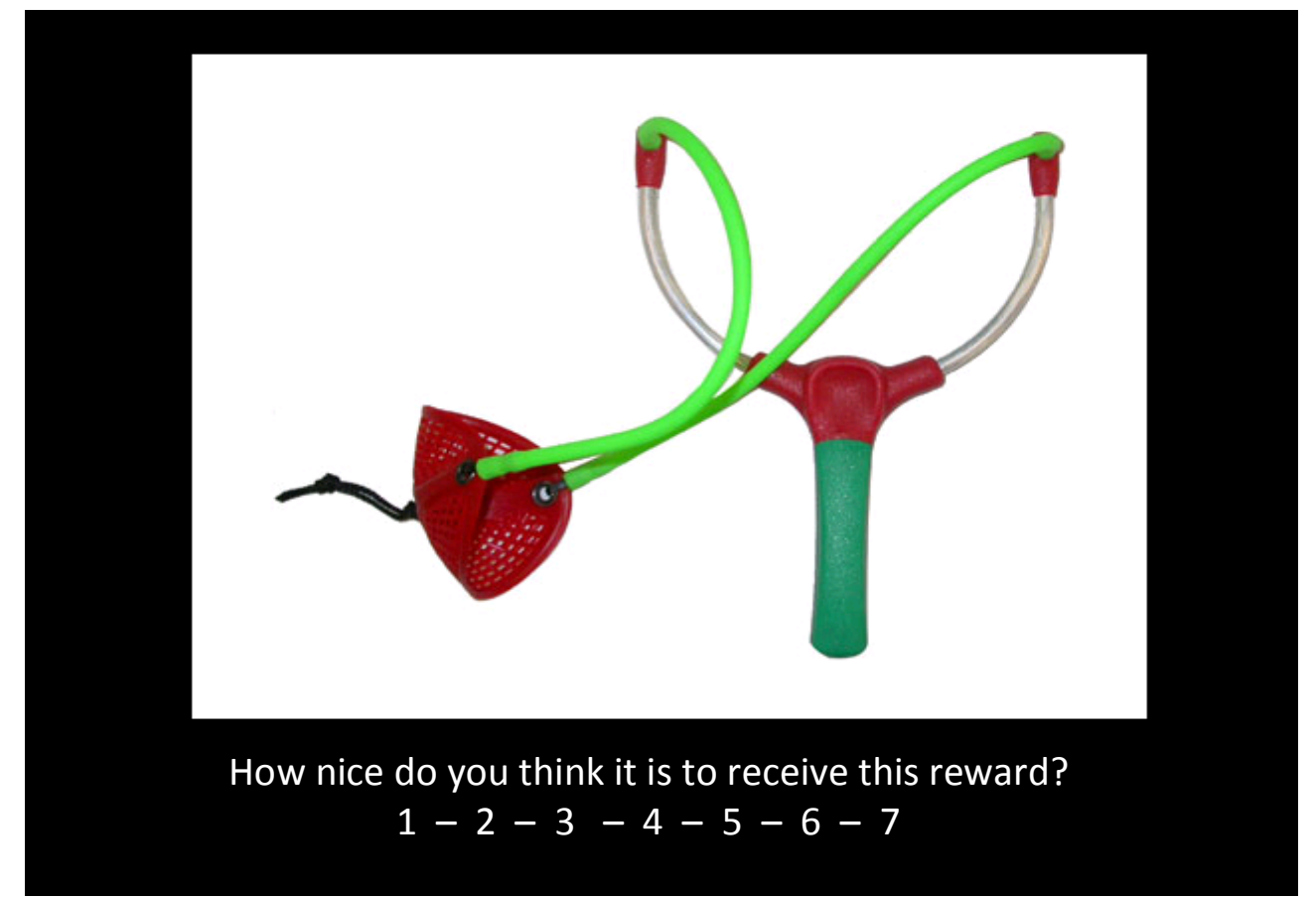

Fig. 1 Example of a trial in the first task

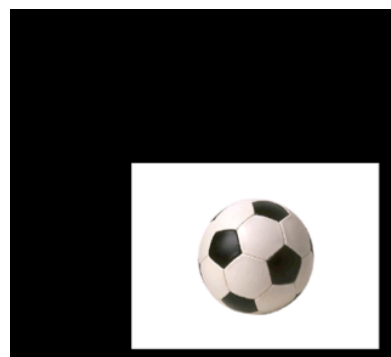

1 week

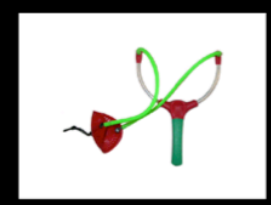

now

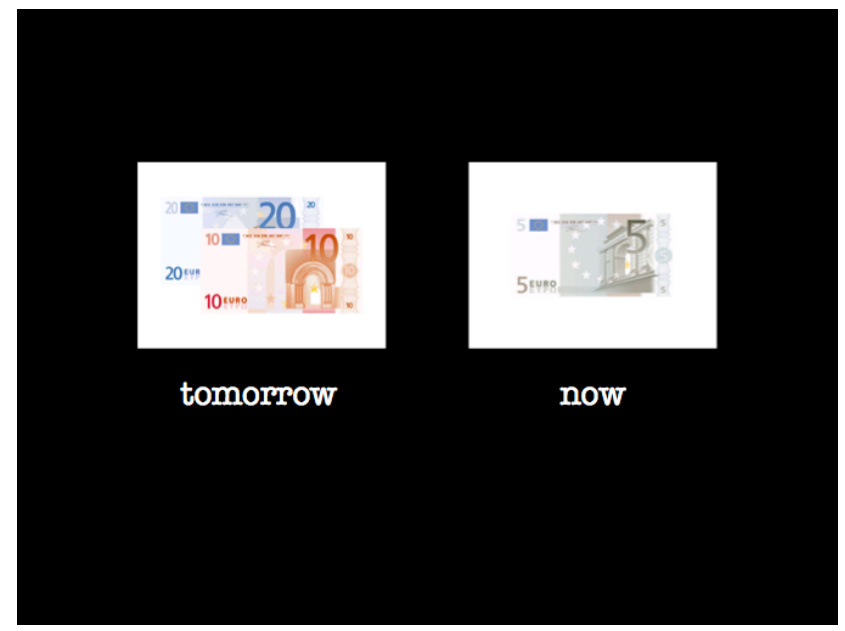

Fig. 2 Example of a trial for the material rewards and monetary TD tasks. Instruction: Which reward do you prefer, taking the delay into account? 


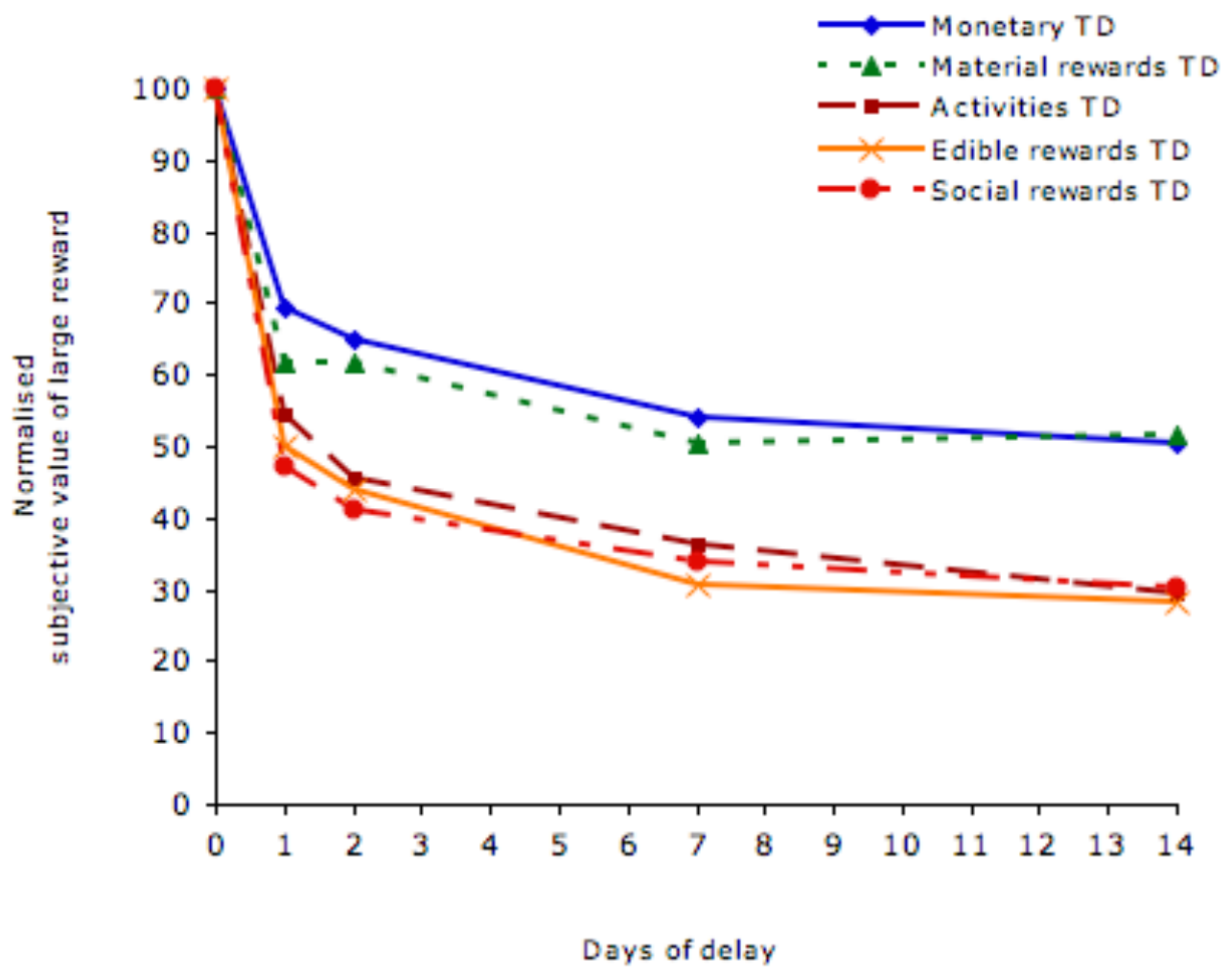

Fig. 3 TD slopes of the different TD tasks

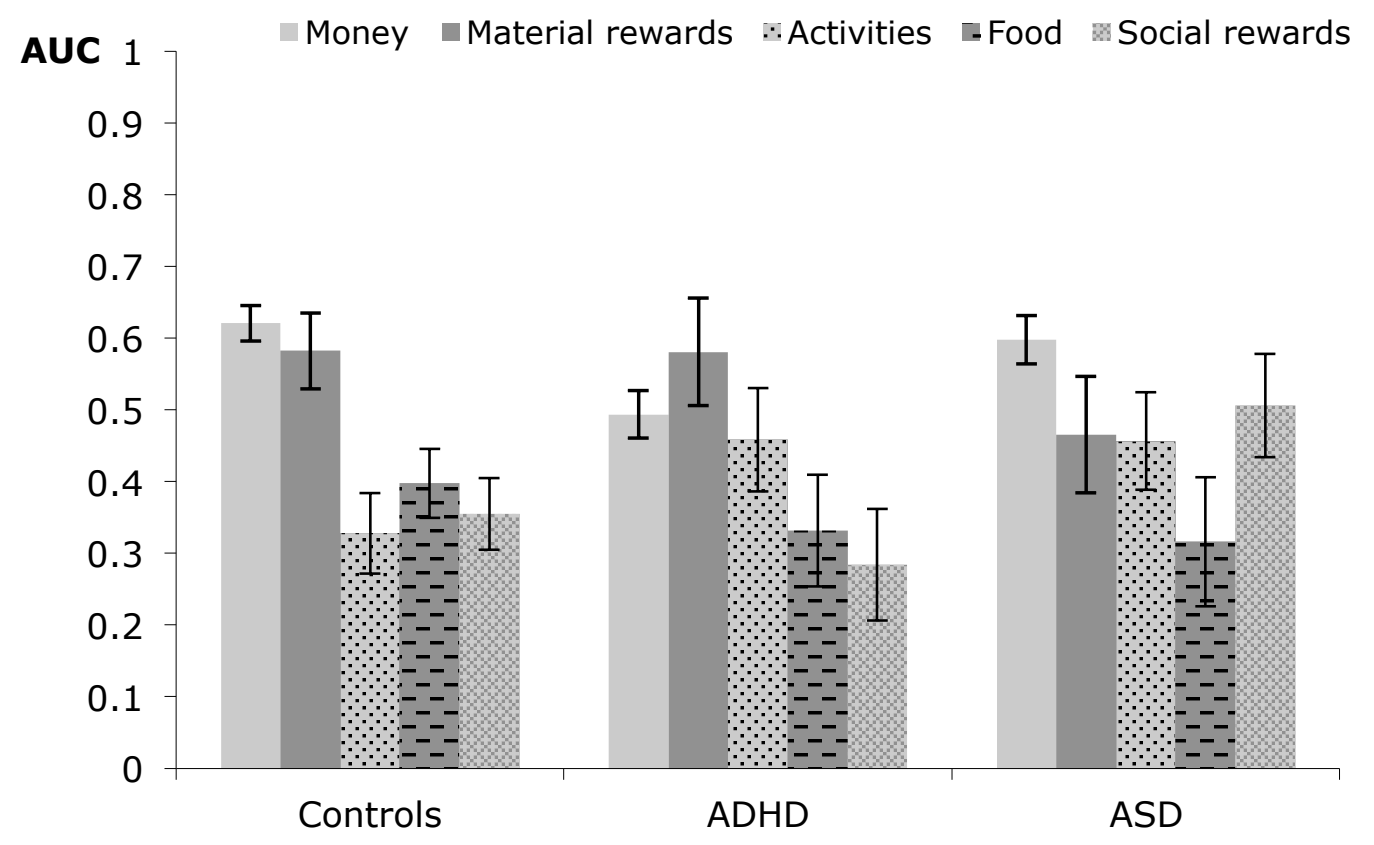

Fig. 4 AUC values of the different TD tasks 
Table 1. Descriptive characteristics of the sample

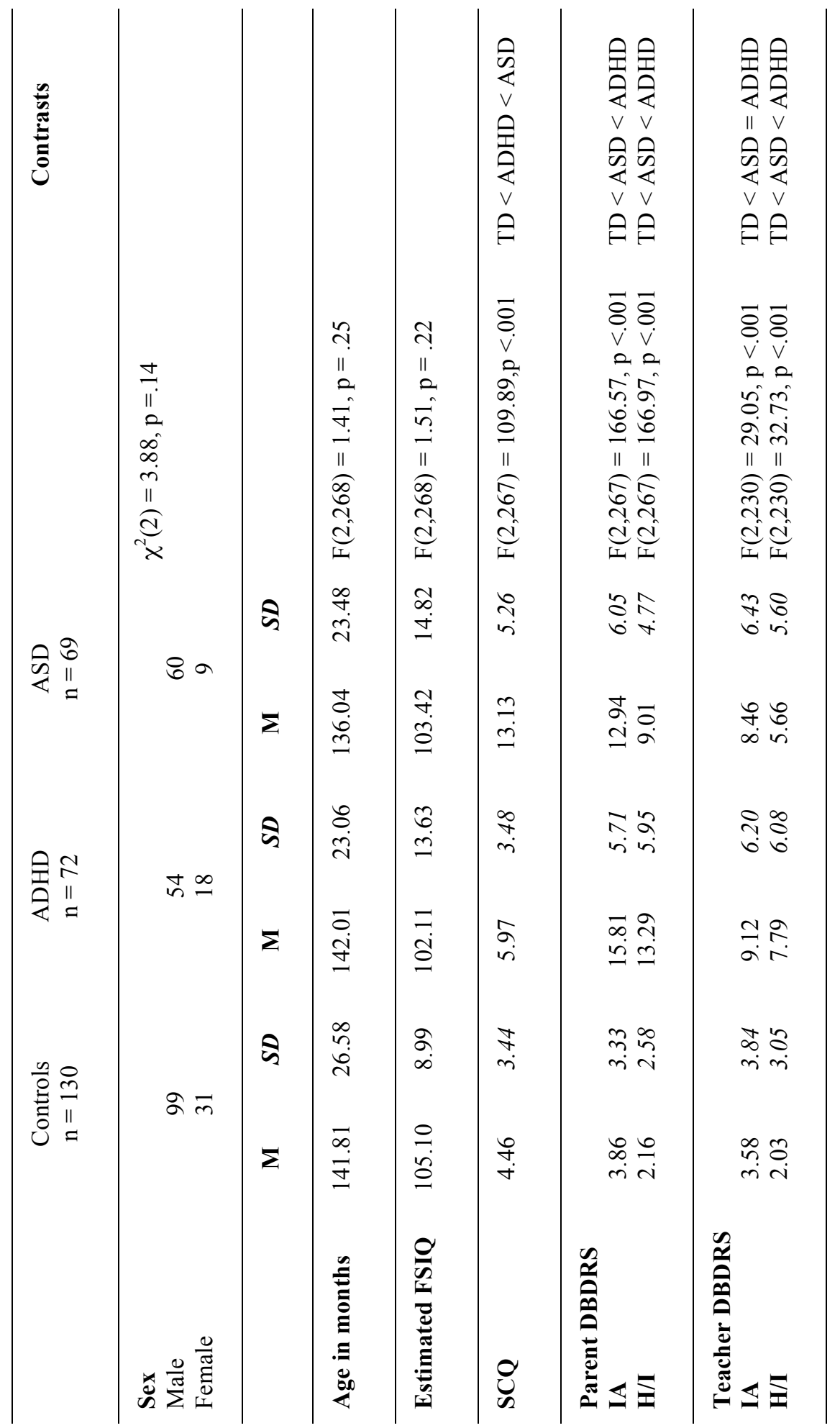

Note: FSIQ: full scale intelligence quotient, SCQ: Social Communication Questionnaire, DBDRS: Disruptive Behavior Disorder rating scale, IA: inattention subscale scores, H/I: hyperactive/impulsive subscale scores 
Table 2. Data of one participant on the monetary TD task, example of the calculation of subjective values of the delayed rewards

\begin{tabular}{llllll}
\hline \multicolumn{7}{c}{ Delay to large reward $(30 \epsilon)$} \\
\hline Immediate reward & \multicolumn{5}{c}{ The day after } \\
& Now & Tomorrow & tomorrow & 1 week & 2 weeks \\
\hline 0 euro & DDDD & DDDD & DDII & DDDD & DDDI \\
5 euros & DDDD & DIII & IIII & DDII & IIII \\
10 euros & DDDD & DIII & IIII & DDII & IIII \\
20 euros & DDDD & DIII & DIII & IIII & IIII \\
30 euros & DDDD & DDII & DDII & IIII & IIII \\
& & & & & \\
\hline Switch points - ascending & 30 & 3.33 & 0 & 5 & 1.66 \\
Switch points - descending & 30 & 30 & 30 & 10 & 1.66 \\
\hline \multirow{2}{*}{ Subjective value } & & & & & \\
\end{tabular}

Note: D: preference for the large delayed reward; I: preference for the immediate reward. For each delay, the subjective value is the mean of the ascending switch point where the choice preference switches from " $\mathrm{D}$ " to "I" and the descending switch point where the choice preference switches from "D" to "I".

Table 3. Mean AUC and standard deviations for the TD tasks

\begin{tabular}{lccccccccc}
\hline & \multicolumn{3}{c}{ Controls } & \multicolumn{3}{c}{ ADHD group } & \multicolumn{3}{c}{ ASD group } \\
\cline { 2 - 10 } AUC values & $\mathbf{M}$ & SD & $\mathbf{n}$ & $\mathbf{M}$ & SD & $\mathbf{n}$ & $\mathbf{M}$ & SD & $\mathbf{n}$ \\
\hline Monetary reward & .62 & .27 & 130 & .49 & .31 & 72 & .60 & .28 & 69 \\
Alternative rewards & & & & & & & & & \\
- Activities & .33 & .27 & 23 & .46 & .29 & 14 & .46 & .27 & 16 \\
- Material reward & .58 & .27 & 26 & .58 & .31 & 13 & .47 & .34 & 11 \\
- Edible reward & .40 & .26 & 31 & .33 & .25 & 12 & .32 & .18 & 9 \\
- Social reward & .35 & .28 & 29 & .28 & .25 & 12 & .51 & .22 & 14 \\
\hline
\end{tabular}

